\title{
Imparting hydrophobic functionalities to copper surface using laser ablation and water emulsion of surfactants
}

\author{
Sergey Grigoriev ${ }^{1}$, Evgeniy Trushin ${ }^{1,}{ }^{*}$, Alena Likhaeva ${ }^{1}$, Alexander Volkov ${ }^{1}$, Marat Dasaev $^{1}$ \\ ${ }^{1}$ National research university «MPEI», Moscow, Russia
}

\begin{abstract}
Wettability is one of the main characteristics of a surface and reflects the ability of a liquid to flow over a solid surface. Using hydrophobic functional surfaces in evaporator and condenser of heating plants to intensify heat exchange processes will increase efficiency and, consequently, reduce the metal consumption and weight-size parameters of this equipment. This will result in reduced energy consumption and lower production and operating costs. This paper presents an analysis of methods for creating hydrophobic surfaces. The fabrication of hydrophobic copper surface of experimental samples by creating multimodal relief in form of $50 \mu \mathrm{m}$ grid by laser ablation process and subsequent reduction of surface energy of material using water emulsion of octadecylamine is grounded and described. The fluence was varied from 5 to $900 \mathrm{~J} / \mathrm{cm}^{2}$ when modifying the surface using laser station. The dependence of the effect of laser fluence on water contact angle value is presented. Following the study, the optimum laser irradiation parameters for the surface of experimental samples were determined, at which the maximum value of water contact angle of $146.61^{\circ}$ and the minimum value of roll-off angle of $13.5^{\circ}$ were obtained.
\end{abstract}

\section{Introduction}

One of the strategic objectives in the energy industry is to improve the reliability and efficiency of heat exchange equipment in heating plants. Thus, phasechange apparatuses (in which the heat transfer agent boils or condenses) are widely used in various heating equipment not only in traditional power engineering, but also in the use of renewable energy sources (RES).

Heat pumps, refrigeration machines and turbines based on low boiling point fluids (LBPF) are widely used in the RES-based energy supply of consumers. Such equipment uses an evaporator and condenser as the main heat exchangers, which are highly metal consuming and have considerable weight-size parameters (Fig. 1). Intensification of heat exchange processes in evaporator and condenser will allow to increase efficiency and, as a consequence, to reduce metal consumption, weight and dimensions of this heat exchangers.

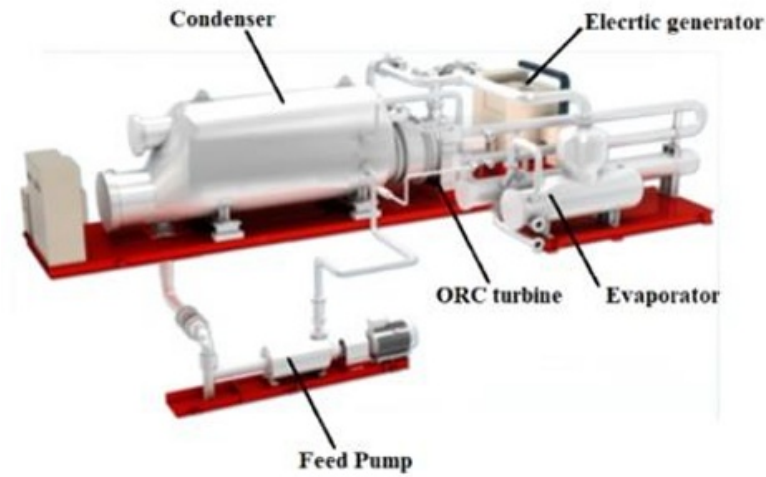

Fig. 1. ORC unit by TURBODEN [1].

The current approach is to use porous coatings, finning, knurling, dimpled surfaces, inclined surfaces, etc. to intensify the heat transfer during boiling and condensation [2].

Analysis of various studies shows that the most promising way of intensification of boiling and condensation processes is the use of hydrophobic functional surfaces. Thus, research work [3] studied free convection and heat transfer of evaporating droplets on initial and hydrophobic substrate made of aluminiummagnesium alloy AlMg6. The surface of the experimental sample was made hydrophobic by laser ablation process followed by coating with nanofluid (aqueous solution of $\mathrm{SiO}_{2}$ silicon dioxide), resulting in a water contact angle of $155-160^{\circ}$. The study determined 
that the heat transfer coefficient for the hydrophobic surface is $25-30 \%$ higher than for the initial substrate.

The authors of [4] have developed a fast and simple process for fabrication of silicon based thin film coating. This technique makes it possible to achieve a hydrophobic surface state. In this case the value of the water contact angle is 105 degrees. Experimental studies were carried out on a water vapour condensation unit. Fig. 2 shows the schematic diagram of this unit. The experimental results indicated that the said silicon coating on copper tube surface, as compared to untreated copper tube, contributes to increase the heat transfer coefficient during condensation by $50 \%$. It was also observed that the addition of silicon oil to synthetic film can significantly increase the evaporation rate of water droplets from the surface, while the surface wettability remains the same.

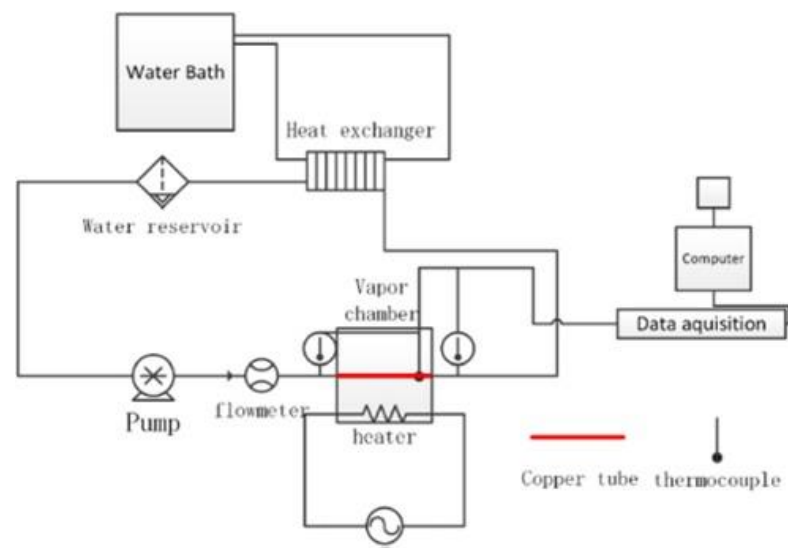

Fig. 2. Schematic view of water vapour condensation plant [5].

In research work [5] a comparative analysis of heat transfer coefficient values in vertical plate heat exchangers made of brass 464 in the initial state and with PTFE-based hydrophobic coating was carried out. Analysis of the results of experiments carried out at identical operating parameters indicates that the use of hydrophobic coatings contributes to increase in the heat transfer coefficient by 4 times, compared with the surface in the initial state.

In addition, hydrophobic surfaces contribute to reducing the rate of deposit formation and corrosion processes [6].

The scientific community has identified the basic conditions that must be met in order to create superhydrophobic surfaces [7]:

- creation of multimodal relief on the surface of material;

- reducing the surface energy of the solid surface of material.

There are many ways to create hydrophobic metal surfaces. Electrodeposition of nanoparticles, chemical vapour deposition and etching, plasma treatment, etc. are the most common. These methods are very expensive, complicated and have low mechanical properties of the treated surface structure. For example, hydrophobic surfaces made using some of the above- mentioned techniques are very brittle and the coating may delaminate [8].

The modification of surfaces of various construction materials using laser equipment is currently being actively developed. This method does not have any of the previously mentioned disadvantages and allows the creation of a multimodal ordered topography with repeatable geometric characteristics. This makes it possible to achieve high values of the water contact angle. However, the construction material surface after the laser ablation process has hydrophilic properties due to formation of metal oxides on it [9].

Surface energy can be reduced by storing the lasermodified sample in the air for 10-30 days [10-12] (Fig. 3 ), by creating a surfactant layer on its surface [8] and by other ways. a)

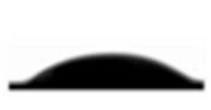

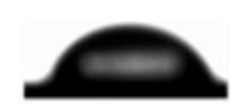

b)

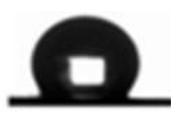

c)
Fig. 3. Images of water droplet shape on modified surface on day 1 (a), day 7 (b) and day 20 (c) after laser ablation processing.

The duration of imparting hydrophobic properties to the surface when stored in ambient air is one of the reasons preventing mass production for industrial applications. It should be noted that the layer formed by adsorption of airborne organic compounds on lasertreated surface is easily removed from surface by exposure to liquid media. As a consequence, there is deterioration in the non-wettability properties of the surface [14].

The authors of [12] used 50×50 mm mirror-polished stainless steel $316 \mathrm{~L}$ plates as experimental samples. Imparting hydrophobic functionalities to metal surface was carried out using $1030 \mathrm{~nm}$ laser equipment with the following source parameters: scan speed was $100 \mathrm{~mm} / \mathrm{s}$, rep rate was $100 \mathrm{kHz}$ and pulse duration was 10 picoseconds. The value of water contact angle, which increased between 77 and 154 degrees, was measured after exposing the samples to ambient air for 30 days in order to sorb the organic substances on their surface.

In research work [15], experimental samples made of aluminium AA2024 were coated with a perfluoropolyether compound as a hydrophobic agent after laser texturing of the surface. The sample surface in the initial state demonstrated hydrophilic properties and the value of the water contact angle was $59 \pm 2^{\circ}$. After surface modification, the water contact angle was $166 \pm 2^{\circ}$.

In the present study, imparting hydrophobic functionalities to the copper surface of experimental samples was performed by using a laser station to fabricate a grid pattern with lines equidistant by $50 \mu \mathrm{m}$, followed by formation of surfactant layers. The influence of laser fluence on surface wettability properties of the samples was analysed.

\section{Description of research methods}


To study the change of functional surface, plates with dimensions $40 \times 40 \mathrm{~mm}$ and thickness of $1 \mathrm{~mm}$ made of flat copper M1t were used as experimental samples.

After the copper plates were made, the surface was cleaned of various contaminants using ethyl alcohol and then washed with distilled water to remove solvent residues.

The multimodal relief was formed on the surface of experimental samples using the FMark-20 NS-FB laser station (Fig. 4) with the following technical specifications:

- $1064 \mathrm{~nm}$ ytterbium fibre laser;

- rep rate ranges from 20 to $100 \mathrm{kHz}$;

- maximum output power is $20 \mathrm{~W}$;

- scan speed up to $10 \mathrm{~m} / \mathrm{s}$;

- pulse duration ranges from 4 to $200 \mathrm{~ns}$;

- spot size is $59 \mu \mathrm{m}$.

The laser beam is focused on the treated surface using MS-II-10 dual axis deflection system (RAYLASE AG, Germany). Linos lens with focal length of $163 \mathrm{~mm}$ and scan field of $110 \times 110 \mathrm{~mm}$ was used for laser scanning.

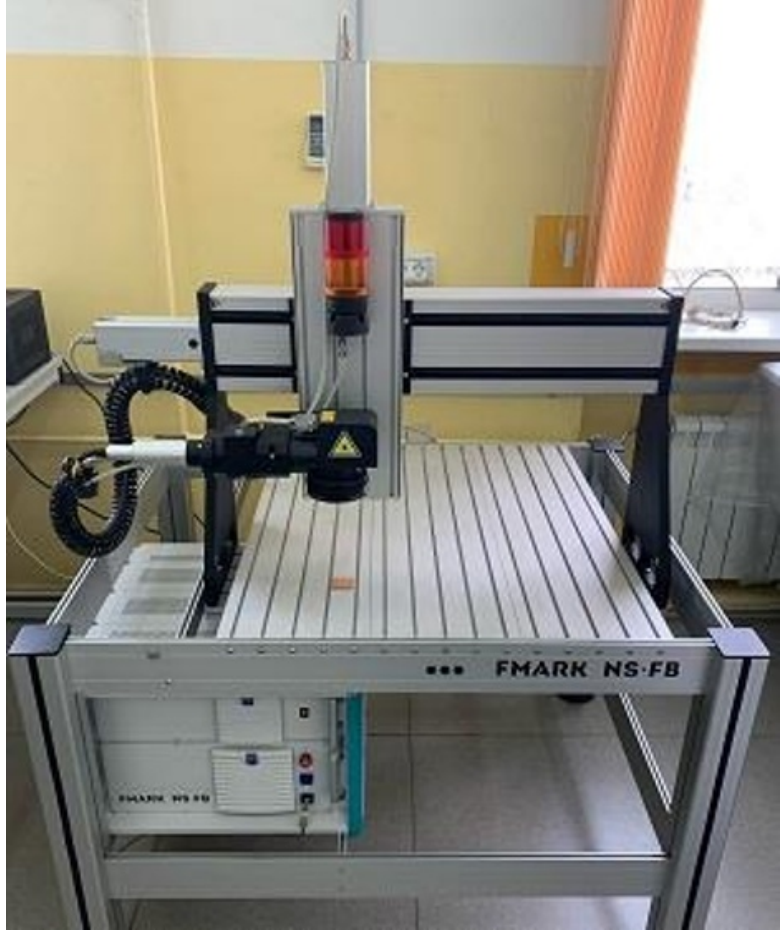

Fig. 4. Photo: Laser station FMark-20 NS-FB.

To produce a gridded relief structure, the laser beam moved along the sample surface along mutually perpendicular lines spaced at equal distance of $50 \mu \mathrm{m}$ from each other. During the treatment of experimental samples surfaces the laser fluence was varied in the range from 5 to $900 \mathrm{~J} / \mathrm{cm}^{2}$, which corresponds to the following parameters: laser output power was $12,20 \mathrm{~W}$, rep rate was $20,100 \mathrm{kHz}$, and scan speed varied in the range from 42 to $5555 \mathrm{~mm} / \mathrm{s}$. Laser radiation parameters for each of experimental samples are presented in Table 1.

Table 1. Laser fluence for each experimental sample.

\begin{tabular}{|c|c|c|c|c|}
\hline Sample No. & Laser output power, $\mathrm{W}$ & Rep rate, $\mathrm{kHz}$ & Scan speed, $\mathrm{mm} / \mathrm{s}$ & Laser fluence, $\mathrm{J} / \mathrm{cm}^{2}$ \\
\hline 1 & 12 & 20 & 5555,1 & 5 \\
\hline 2 & 12 & 20 & 2340,9 & 10 \\
\hline 3 & 12 & 20 & 914,9 & 25 \\
\hline 4 & 20 & 20 & 835,7 & 50 \\
\hline 5 & 20 & 20 & 386,7 & 100 \\
\hline 6 & 20 & 20 & 255,1 & 150 \\
\hline 7 & 20 & 20 & 190,7 & 200 \\
\hline 8 & 20 & 20 & 152,3 & 250 \\
\hline 9 & 20 & 20 & 126,8 & 300 \\
\hline 10 & 20 & 20 & 108,6 & 350 \\
\hline 11 & 20 & 20 & 95,0 & 400 \\
\hline 12 & 20 & 20 & 84,5 & 450 \\
\hline 13 & 20 & 20 & 76,0 & 500 \\
\hline 14 & 20 & 20 & 63,3 & 600 \\
\hline 15 & 20 & 20 & 54,3 & 700 \\
\hline 16 & 20 & 20 & 47,5 & 800 \\
\hline 17 & 20 & 20 & 42,2 & 900 \\
\hline
\end{tabular}

Molecular surfactant layers were formed on the surface of textured samples using a designed and constructed unit. Fig. 5 shows the schematic diagram of this unit. 


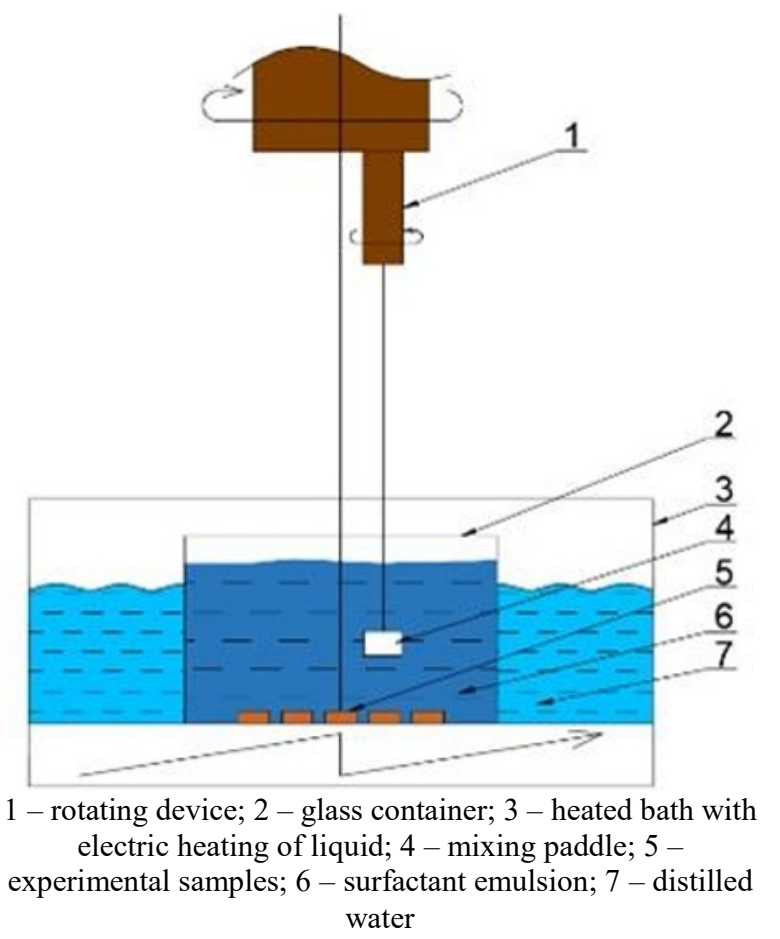

Fig. 5. Schematic diagram of unit for formation of molecular layers of surfactants on surface of experimental samples.

This unit consists of a laboratory heated bath (3) with electric heating of liquid (7), in which a glass container (2) is immersed to form molecular layers of surfactant, as which octadecylamine (ODA) was used. At the bottom of container the experimental samples (5) are evenly placed in horizontal position, then paddle (4) is immersed and rotated planetary and multidirectionally by rotating device (1), thus providing uniform mixing of ODA emulsion (6).

In the experiment, a bath is heated by means of electric heating elements, then a container filled with 3 $\mathrm{L}$ of distilled water is immersed in the heated bath. When the water temperature in container reaches $80^{\circ} \mathrm{C}$ [16], $9 \mathrm{mg}$ of octadecylamine is added and actively stirred to a homogeneous emulsion using rotating device. The experimental samples are then lowered to the bottom of container with emulsion and the mixing paddle is immersed. The paddle is rotated both about its own axis and about the axis of the glass container, coaxial to rotating device, for 30 minutes. After this time, the samples are removed from emulsion and air dried for 24 hours until the treated surface is completely dry. Fig. 6 shows images of surface of experimental samples in their initial state and after modification using the laser station and aqueous emulsion of ODA.

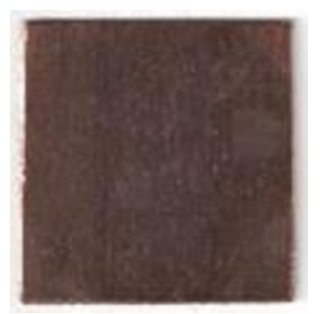

a)

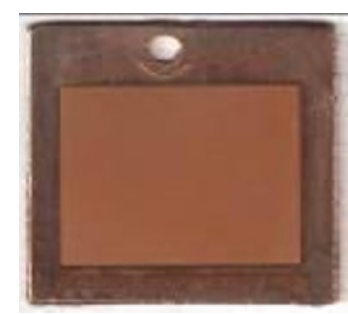

b)

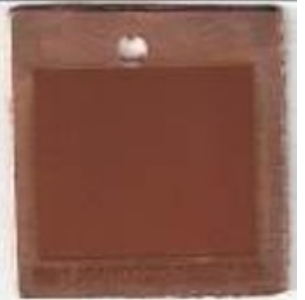

c)

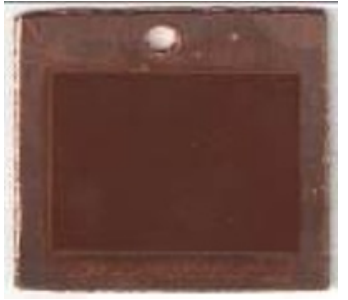

d)
Fig. 6. Images of experimental samples with initial (a) and modified surface at laser fluence of 50 (b), 250 (c) и 900 (d) $\mathrm{J} / \mathrm{cm}^{2}$.

Fig. $6(b-d)$ shows that the experimental samples after laser ablation and formation of molecular layers of ODA have colour differences. This is primarily due to the fact that the geometric characteristics of relief, such as side protrusion height and channel depth, increases as the laser fluence increases.

After the modified sample surface had completely dried, the values of water contact angle and roll-off angle were determined using an OCA 20 device (Fig. 7) (enters as part of large-scale research facilities «Hydroshock test rig Erosion-M») and 5 and $30 \mu \mathrm{l}$ drops of distilled water, respectively (Fig. 8). Water contact angle and roll-off angle were measured on three different areas of the experimental sample surface modified with laser station and ODA aqueous emulsion to improve the accuracy of the results obtained The average value of water contact angle and roll-off angle was then calculated.

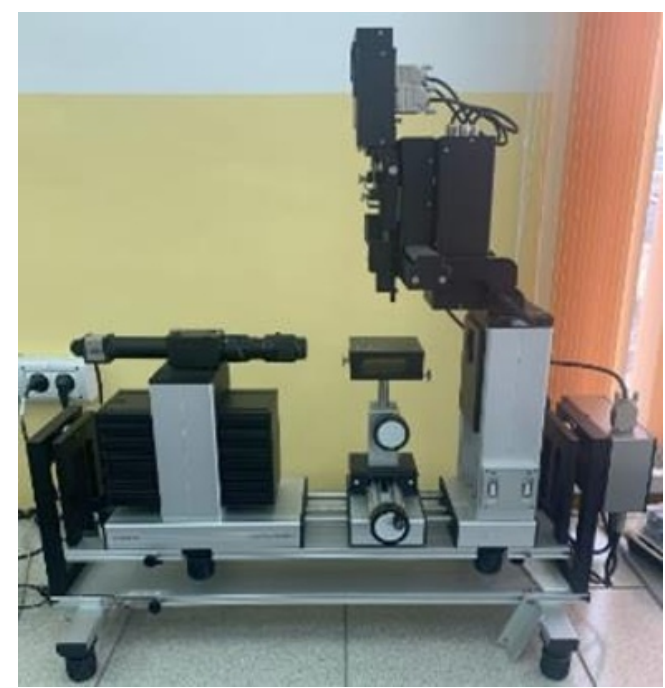

Fig. 7. Photo: OCA 20 device for measuring water contact angle and roll-off angle.

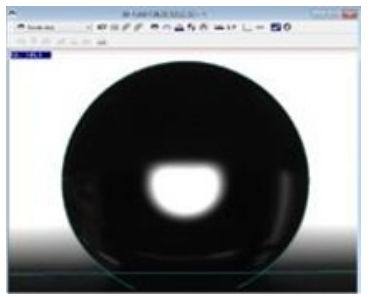

a)

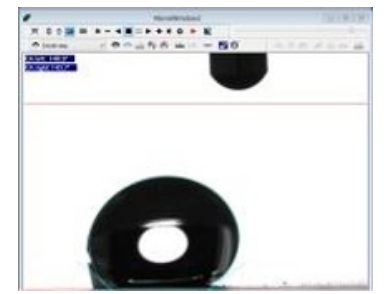

b) 
Fig. 8. Photo: Measuring water contact angle (a) and roll-off angle (b).

The surface of experimental samples in their initial state and after relief formation was also examined using SEM Tescan MIRA 3 (Fig. 9). Fig. 9 demonstrates that the laser ablation process has fabricated multimodal ordered microrelief with repeatable geometric characteristics.

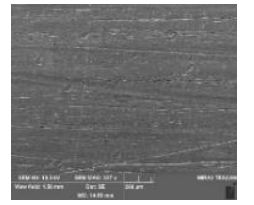

a)

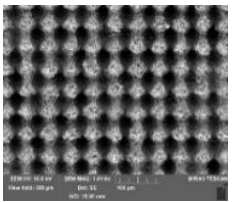

b)

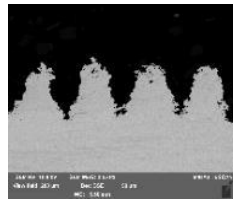

c)
Fig. 9. Images of initial state (a), modified state (b) and micrographic specimen (c) of copper surface.

\section{Results obtained}

The copper surface in its initial state demonstrates hydrophilic properties and the values of the water contact angle and roll-off angle are $70.16^{\circ}$ and $77.53^{\circ}$, respectively. Immediately after laser texturing, all experimental samples achieve a hydrophilic surface state. The modification of the experimental samples using laser ablation process and ODA aqueous emulsion resulted in all surfaces becoming hydrophobic and water contact angle ranging from 102.9 to 146.6 degrees.

The effect of laser fluence on water contact angle was studied (Fig. 10). A surface modified solely with octadecylamine aqueous emulsion at similar parameters was chosen as the initial point at which the laser fluence was zero. The value of the water contact angle for this surface was $104^{\circ}$.

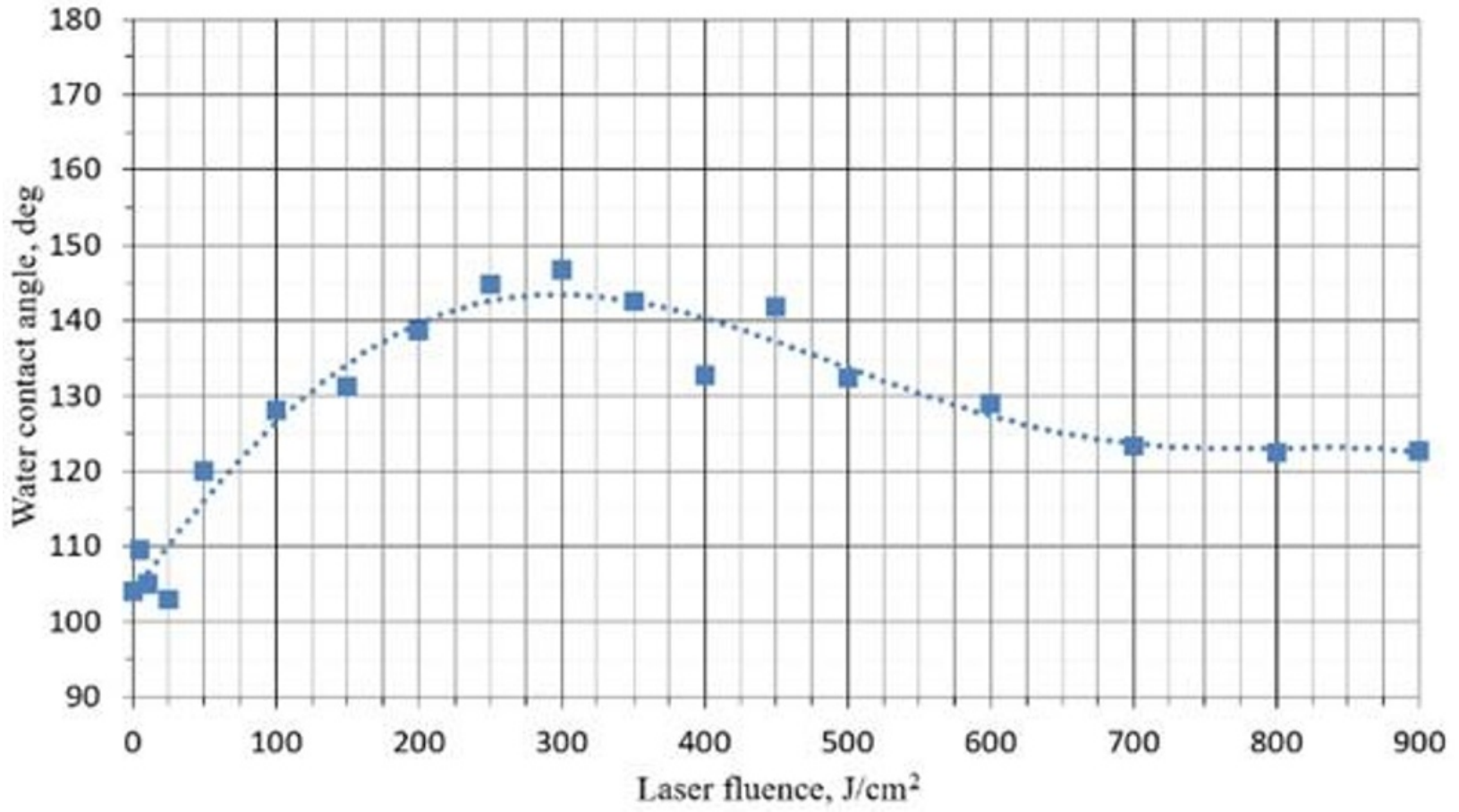

Fig. 10. Dependence of water contact angle on laser fluence.

Fig. 10 shows that increasing in laser fluence from 0 to $300 \mathrm{~J} / \mathrm{cm}^{2}$ leads to an increase in the water contact angle. However, a further increase in the laser fluence leads to a decrease in the water contact angles.

The maximum value of the water contact angle of $146,61^{\circ}$ was obtained at a laser fluence of $300 \mathrm{~J} / \mathrm{cm}^{2}$. The value of the roll-off angle for this surface is $13.5^{\circ}$. This value is the minimum for the experimental samples considered.

\section{Conclusions}

In this work, a grid pattern with lines equidistant by 50 $\mu \mathrm{m}$ was created on the copper sample surface using a laser station, followed by the formation of ODA layers to reduce the surface energy of material.
Analysis of effect of laser fluence on water contact angle (Fig. 10) indicates that the maximum values of water contact angle are reached at $250,300 \mathrm{~J} / \mathrm{cm}^{2}$.

The surface of experimental sample No. 9 (Table 1) with a minimum roll-off angle of $13.5^{\circ}$ and maximum water contact angle of $146.61^{\circ}$ was obtained at laser fluence of $300 \mathrm{~J} / \mathrm{cm}^{2}$.

The investigation was carried out within the framework of the project "Efficiency improvement of low boiling point fluid plants based on use of biphilic heat exchange surfaces" with the support of a grant from NRU "MPEI" for implementation of scientific research programs "Energy", "Electronics, Radio Engineering and IT", and "Industry 4.0, Technologies for Industry and Robotics in 2020-2022. 


\section{References}

1. Official website of TURBODEN S.p.A. // ORC system. URL: https://www.turboden.com/ ( access date: 10.03.2021).

2. B.V. Dzyubenko, Yu.A. Kuzma-Kichta, A.I. Leontiev, I.I. Fedik, L.P. Kholpanov. Intensification of heat and mass transfer on macro-, micro- and nano-scale. - Moscow: FSUE "CRDIATOMINFORM" (2008), with a color tab.

3. S.Y. Misyura. Heat Transfer and Convection of Evaporating Sessile Droplets in Transition from Superhydrophilic to Superhydrophobic Structured Wall: Optimization of Functional Properties // International Communications in Heat and Mass Transfer, Vol. 112, (2020). P. 104474.

4. X. Quan, S. Chen, J. Li, P. Cheng. Enhanced dropwise condensation by oil infused nano-grass coatings on outer surface of a horizontal copper tube // International Communications in Heat and Mass Transfer, Vol. 91, (2018). pp. 11-16.

5. R. Lara Jorge, Mark T. Holtzapple. Experimental investigation of dropwise condensation on hydrophobic heat exchangers part I: Dimpled-sheets; Desalination 278, (2011), pp. 165-172.

6. A.V. Ryzhenkov, M.R. Dasaev, S.V. Grigoriev, A.V. Kurshakov, O.V. Ryzhenkov, M.V. Lukin. The effect of hydrophobicity on hydraulic resistance during transportation of fluid media // International Journal of Emerging Trends in Engineering Research, Vol. 8, (2020), pp. 195-202.

7. A.V. Ryzhenkov, M.R. Dasaev, S.V. Grigoriev, A.V. Kurshakov, O.V. Ryzhenkov, M.V. Lukin. Hydrophobic brass surfaces created by means of multi-scale relief // International Journal of Mechanical Engineering and Technology, Vol. 9, (2018). pp. 58-70.

8. H. Yan, M.R.B.A. Rashid, S.Y. Khew, F. Li, M. Hong. Wettability transition of laser textured brass surfaces inside different mediums // Applied Surface Science, Vol. 427, (2018). pp. 369-375.

9. A. Samanta, Q. Wang, S.K. Shaw, H. Ding. Roles of chemistry modification for laser textured metal alloys to achieve extreme surface wetting behaviors // Materials \& Design, Vol. 192, (2020). P. 108744.

10. L. Jiao, Z. Chua, S. Moon, J. Song, G. Bi, H. Zheng. Femtosecond Laser Produced Hydrophobic Hierarchical Structures on Additive Manufacturing Parts // Nanomaterials, Vol. 8, (Aug 2018). P. 601.

11. O. Raimbault, S. Benayoun, K. Anselme, C. Mauclair, T. Bourgade, A.M. Kietzig, P.L. GirardLauriault, S. Valette, C. Donnet. The Effects of Femtosecond Laser-textured Ti-6Al-4V on Wettability and Cell Response // Materials Science and Engineering: C, Vol. 69, (2016). pp. 311-320.

12. A. Peter, A.H.A. Lutey, S. Faas, L. Romoli, V. Onuseit, T. Graf. Direct Laser Interference Patterning of Stainless Steel by Ultrashort Pulses for Antibacterial Surfaces // Optics \& Laser Technology, Vol. 123, (2020). P. 105954.

13. G.B. Shirsath, K. Muralidhar, R.G.S. Pala, J. Ramkumar. Condensation of Water Vapor
Underneath an Inclined Hydrophobic Textured Surface Machined by Laser and Electric Discharge // Applied Surface Science, Vol. 484, (2019). pp. 9991009.

14. L.B Boinovich, A.M. Emelyanenko, K.A. Emelyanenko, A.G. Domantovsky, A.A. Shiryaev. Comment on "Nanosecond laser textured superhydrophobic metallic surfaces and their chemical sensing applications" by D. V. Ta, A. Dunn, T.J. Wasley, R.W. Kay, J.E. Stringer, P.J. Smith, C. Connaughton, J. D. Shephard (Appl. Surf. Sci. 357 (2015) 248-254) // Applied Surface Science, Vol. 379, (2016). pp. 111-113.

15. V. Vercillo, J.T. Cardoso, D. Huerta-Murillo, S. Tonnicchia, A. Laroche, J.A.M. Guillén, J.L. Ocaña, A.F. Lasagni, E. Bonaccurso. Durability of Superhydrophobic Laser-treated Metal Surfaces under Icing Conditions // Materials Letters: X, Vol. 3, (2019). P. 100021.

16. Patent 2439204 C1, Russian Federation, IPC C23F 11/14. Method of protection of hydraulic systems surfaces against corrosion and deposit accumulation: № 2010124248/02: applied for. 16.06.2010: publ. 10.01.2012 / V.A. Ryzhenkov, A.V. Kurshakov, I.P. Anakhov, O.V. Kalakutskaya - 4 p. 